\title{
Molecular typing of Mycobacterium tuberculosis complex isolated from pulmonary tuberculosis patients in central Ethiopia
}

Zufan Bedewi ${ }^{1,2,3}$, Adane Worku', Yalemtsehay Mekonnen², Getnet Yimer, Girmay Medhin', Gezahegne Mamo4, Rembert Pieper ${ }^{6}$ and Gobena Ameni ${ }^{1 *}$

\begin{abstract}
Background: Identification of the types of strains of Mycobacterium tuberculosis (M. tuberculosis) complex causing tuberculosis (TB) could contribute to TB control program of specific geographic region as well as it could add knowledge onto the existing literature on TB worldwide. The objective of the present study was to identify the species and strains of M. tuberculosis complex causing pulmonary tuberculosis in central Ethiopia.

Methods: A health institution- based cross-sectional study was conducted on 338 smear positive TB cases visiting three hospitals between October 2012 and September 2013. Morning and spot sputum samples were collected before the starting of treatment regimens. Thus, a total of 338 pooled sputum samples collected from these cases. Samples were cultured on Löwenstein Jensen media and the isolates were identified by the region of difference (RD) 9 based polymerase chain reaction (PCR) and spoligotyping.

Result: Of the total isolates $98.6 \%$ of the isolates were identified to be $M$. tuberculosis while the remaining $1.4 \%$ were identified as M. africanum. Further, typing of $M$. tuberculosis using spoligotyping lead to the identification of 90 different strains of $M$. tuberculosis. Of these strains, 32 were clustered consisting of more than one isolate while the remaining 58 strains were unique consisting of single isolate. Thus, $79.3 \%(223 / 281)$ of the isolates were found in the clustered while only $20.6 \%$ (58/281) of the strains were unique. Forty-five of the spolgotyping patterns were registeredin the SITVIT2 or SpoIDB4 database in while the remaining 45 were notfound in the database and hence were orphan strains. The dominant strains were SIT53, SIT149, and SIT54, consisting of 43, 37 and 34 isolates, respectively. Classification of the spoligotype patterns using TB-insight RUN TB-Lineage showed that 86.8, 6.4, 5, 1. $4 \%$ ofthe isolatesbelonged to the Euro-American lineage, East-African-Indian, Indo-oceanic and M. africanum, respectively.

Conclusion: The identification of clustered and new strains using spolygotyping in present study does not give conclusive finding as spoligotyping has low discriminatory power. Thus, further identification of these isolates using mycobacterial interspersed repetitive unit-variable number tandem repeat (MIRU-VENTR) and or whole genome sequencing (WGS) recommended.
\end{abstract}

Keywords: Diversity of strain, M. tuberculosis, Central Ethiopia

\footnotetext{
* Correspondence: gobena.ameni@aau.edu.et

${ }^{1}$ Aklilu Lemma Institute of Pathobiology, Addis Ababa University, P.O. Box

1176, Addis Ababa, Ethiopia

Full list of author information is available at the end of the article
} International License (http://creativecommons.org/licenses/by/4.0/), which permits unrestricted use, distribution, and reproduction in any medium, provided you give appropriate credit to the original author(s) and the source, provide a link to the Creative Commons license, and indicate if changes were made. The Creative Commons Public Domain Dedication waiver (http://creativecommons.org/publicdomain/zero/1.0/) applies to the data made available in this article, unless otherwise stated. 


\section{Background}

Tuberculosis (TB) is one of an infectious disease, affecting millions of people worldwide. According to the $13^{\text {th }}$ Annual TB Report in 2014, there were 9.6 million new TB cases and 1.5 million TB deaths annually [1]. The spread of human immunodeficiency virus (HIV) and drug-resistant TB have exacerbated the situation.

Ethiopia has high rate of $\mathrm{TB}$ infection and the disease is one of major public health problems in the country [2]. According to a WHO report, the country is one among the world's 22 countries with high TB burden [1]. The annual incidence of new TB cases was estimated to be $207 / 100,000$ and the prevalence of TB in the country was $200 / 100,000$ [1]. The country is one of the 27 high MDR-TB countries; ranked $15^{\text {th }}$ with more than 5000 estimated MDR-TB patients each year $[1,2]$. MDR TB was $1.6 \%$ of new cases and $12 \%$ of re-treatment cases [1].

Effective TB control program requires understanding of its epidemiology including the strains of M.tuberculosiscomplex (MTBC) circulating in the population [3]. Molecular epidemiological studies have been used to provide valuable information onthe spread of tubercle bacilli in outbreaks [4] and also contributed to study the transmission dynamics of TB [5]. Moreover, such studies can help in distinguishing exogenous reinfection from endogenous reactivation [6]. Additionally, molecular epidemiological studiescan be used to identify laboratory cross contamination [7] and to track the geographic distribution and spread of clones, including Multi drug resistant strains [8]. Furthermore, molecular typing has shown the large differences in pathobiological properties of MTBC species [9].

In Ethiopia, TB is a major public health problem and few molecular epidemiological studies have been done so far in some part of the country. While the genetic diversity of $M$. tuberculosis lineages in the country has been investigated [10-13], there is little or no data in some part of the country including this study area. The availability of such information will help study the phylogenetic characteristics of an organism, which in turn will provide new insight into the natural history of M. tuberculosis and Knowledge on the Mycobacterium tuberculosis strains circulating in the country communities is useful for epidemiology, transmission, and is essential in the control of the disease.

Thus, identificationthe types of strains of MTBC causing $\mathrm{TB}$ in a specific geographic region could contribute to the strengthening the TB control program of that specific geographic region, as it can alert personnel in the TB Control Program so that they can monitor the transmission of special strains such as drug resistant and virulent strains. In addition, identificationthe types of strains circulating in a specific geographic region could help in adding additional knowledge on the existing literature on TB worldwide. To our knowledge there is scarcity of information on strains of $M$. tuberculosis of circulating in central Ethiopia. Therefore, the objective of the present study was to identify the species and strains of MTBC causing pulmonary TB in central Ethiopia.

\section{Methods \\ Study area}

This study was performed at three different sites in central Ethiopia. These sites were Woliso and Atat towns and their surroundings in the southwest of Addis Ababa at a distance of $114 \mathrm{~km}$ and $187 \mathrm{~km}$, respectively. The third site was Fiche town and its surrounding in the northwest of Addis Ababa at $115 \mathrm{~km}$. Sample collection was performed at hospitals located at these three sites, namely, St. Lukas, Atat and Fitche hospitals located at Woliso, Atat and Fitche tows, respectively. Sputum samples were collected from smear positive TB cases visiting these three hospitals.

\section{Study design and study subjects}

A health institution-based cross-sectional study was conducted on 338 smear positive TB patients visiting three hospitals, between October 2012 and September 2013. Three consecutive sputum samples (spot, early morning and spot) were collected from each of these 338 smear positive TB patient and pooled together for culturing. Sample collection was performed prior to the beginning of TB treatment. TB patients less than 18 years old excluded from this study. Socio-demographic data of the patients were obtained from the medical records of all patients.

Those samples positive for acid fast bacilli (AFB) by Ziehl-Neelsen (ZN) staining technique were collected labeled and samples from the individual patient pooled together. These sputum samples for culture were stored at $-20^{\circ} \mathrm{C}$ and then transported in a cold box $\left(\right.$ at $\left.+4^{\circ} \mathrm{C}\right)$ to Aklilu Lemma Institute of Pathobiology (ALIPB), Addis Ababa, within a week for culture.

\section{Culture}

Morning and spot sputum samples were collected and processed for culture following the WHO Guideline [14]. Briefly, equal volume of $4 \% \mathrm{NaOH}$ was mixedwith sputum sample and the mixture was centrifuged at $3000 \mathrm{rpm}$ for $15 \mathrm{~min}$ at room temperature. After decanting the supernatant the sediment was neutralized with $2 \mathrm{~N}$ HClusing phenol red as an indicator. Neutralization was achieved when the color of the solution was changed from purple to yellow. Thereafter, $100 \mu \mathrm{l}$ of the suspension was inoculated ontotwo sterile LJ medium slopes (which were 
enriched with either pyruvate or glycerol). The inoculated media were then incubated at $37^{\circ} \mathrm{C}$ in slanted positionfor 1 week and upright position for 4-5 weeks. The growth of the bacteria was read every week until the $8^{\text {th }}$ week of culture.

\section{Preparation of specimens for molecular typing}

Colonies were removed from the surface of LJ medium and suspended in $200 \mu \mathrm{l}$ of sterile double distilled water. Thereafter, the colonies and water were mixed thoroughly and then, the mixture was heated at $80^{\circ} \mathrm{C}$ for $1 \mathrm{~h}$ in water bath. This is followed by centrifugation after which the supernatant was collected and used for amplification [15].

\section{Region of difference (RD) 9-based polymerase chain reaction $(\mathrm{PCR})$}

Identification of $M$. tuberculosis from the other members of $M$. tuberculosis complexspecies was done using RD9-based PCR. RD9-PCRwas performed on heatkilled cells to confirm the presence or absence of RD9 using three primers namely, RD9flankF, RD9 IntR, and RD9flankR. Amplification was done by standard thermo cycler (VWR Thermo cycler, UK). The PCR amplification mixture used consisted of $10 \mu \mathrm{lHotStarTaqMaster} \mathrm{Mix}$ (Qiagen, United Kingdom), 7.1 $\mu$ l distilled water, $0.3 \mu \mathrm{l}$ of each three primers and $2 \mu \mathrm{l}$ of DNA template(heat killed cells), giving a total volume of $20 \mu \mathrm{l}$. The PCR reaction was heatedat $95^{\circ} \mathrm{C}$ for $15 \mathrm{~min}$ after which it was subjected to 35 cycles consisting of $95^{\circ} \mathrm{C}$ for one min, $55^{\circ} \mathrm{C}$ for one minutes, and $72{ }^{\circ} \mathrm{C}$ for one minute. Thereafter, the reaction mixture was maintained at $72{ }^{\circ} \mathrm{C}$ for $10 \mathrm{~min}$ following which the product was removed from the thermocycler and run on agarose gel electrophoresis. For gel electrophoresis, $8 \mu \mathrm{l}$ PCR products was mixed with $2 \mu \mathrm{l}$ loading dye, loaded onto $1.5 \%$ agarose gel and electophoresed at $100 \mathrm{~V}$ and $500 \mathrm{~mA}$ for $45 \mathrm{~min}$. The gel was then visualized using a computerized Multi- Image Light Cabinet (VWR). M. tuberculosis H37Rv, M. bovisbacilleCalmette-Guérin, and water were included as positive and negative controls. Interpretation of the result was based on bands of different sizes, as previously described by Parsons et al. [16].

\section{Spoligotyping}

Isolates that were positive for M. tuberculosis by RD9 PCR were further characterized by spoligotyping following the procedure described by Kamerbeeket al [17] and by observing the instructions of the spoligotype kit supplier (Ocimum Biosolutions Company, Iisselstein, and the Netherlands). The direct repeat (DR) region of the isolate was amplified by PCR using oligonucleotide primers (DRa and DRb) derived from the DR sequence [17]. The amplified biotinylated products were hybridized to a set of 43 oligonucleotides covalently bound to a membrane (Animal and Plant Health Agency, Great Britain). Bound fragments were incubated with streptavidinperoxidase conjugate and hybridizing DNA was detected by the enhanced chemiluminescence method, by exposure to X-ray film (Hyperfilm ECL, Amersham) as specified by the manufacturer's instruction. The presence and absence of spacers was visualized on the film as black and white squares, respectively. Characterized strains of $M$. bovisand M. tuberculosis H37Rv were used as positive controls, whereas Qiagen distilled water (Qiagen company, Germany) was used as a negative control.

\section{Use of SpolDB4 and Run TB-Lineage for the identification of strains and lineages}

The results of spoligotyping were converted into octal and binary formats. These binary and octal formats of the strains were entered into query box so that the name of the strains are retrieved from the database if the spoligotype pattern of the strain in question fits the pattern that has already been registered in the SPolDB4 database [18] and at http://www.pasteur-guadeloupe.fr:8081/SITVITDemo/ [10]. If the pattern of the strain in question has not been registered in SPolDB prior to this study, the strain was considered as an orphan. In this study, an isolate is referred to as a colony that was grown on LJ media and found to be AFB-positive after staining with Ziehl Neelsen staining, whereas a strain is an isolate (s) with specific spoligotype pattern. Thus, a strain can consist of a single isolate or several isolates. A strain with a single isolate is termed as a unique strain while a strain with more than one isolate is considered as clustered strain. An

Table 1 Background characteristics tuberculosis patients from whom the M. tuberculosis isolates were obtained

\begin{tabular}{ll}
\hline Background characteristics & Number (\%) of patients \\
\hline Sex & $151(53.7)$ \\
Male & $130(46.3)$ \\
Female & \\
Age, Years & $99(35.2)$ \\
$18-28$ & $72(25.8)$ \\
$29-39$ & $74(26.3)$ \\
$40-60$ & $36(12.8)$ \\
$>50$ & \\
History of anti-tuberculosis treatment & $10(3.6)$ \\
Previously treated & $271(96.4)$ \\
Not previously treated & \\
Region & $134(47.7)$ \\
Woliso & $97(34.5)$ \\
Fiche & $50(17.8)$ \\
Atat &
\end{tabular}




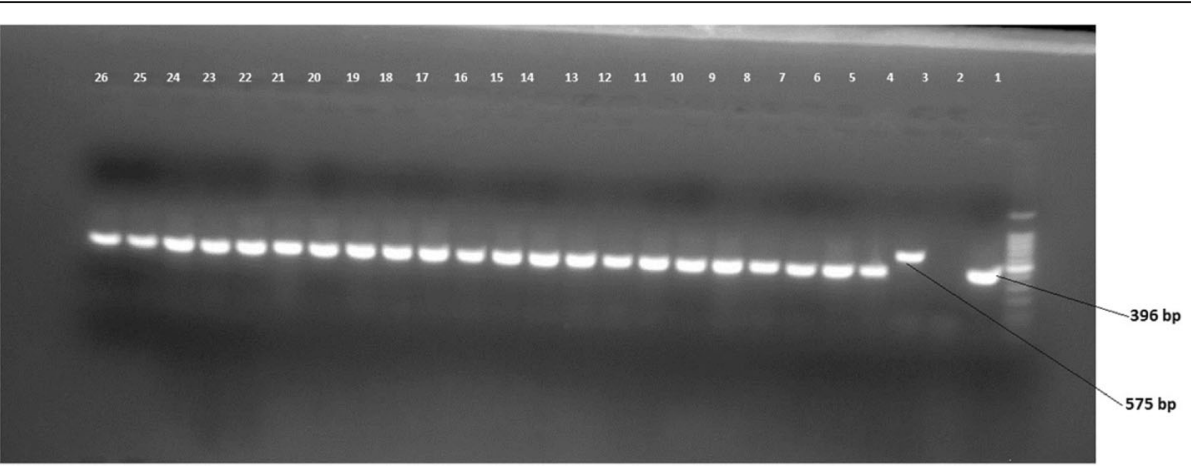

Fig. 1 Picture of gel electrophoresis for RD9 deletion typing of M. tuberculosis isolates. Lane 1 is a ladder, lane 2 is M. Tuberculosis control, lane 3 is Negative control (molecular grade $\mathrm{H} 2 \mathrm{O}$ ), lane 4 is M. bovis control and lane 5-26 is culture isolate of M. Tuberculosis

online tool Run TB-Lineage http://tbinsight.cs.rpi.edu/ run_tb_lineage.html was also used to predict the major lineages using a conformal Bayesian network (CBN) analysis and sub lineage using knowledge based Bayesian network (KBBN).
Statistical analysis

The statistical analysis was performed using STATA software version 12. Descriptive statistics were used to depict the demographic variables. Pearson chi-square was used to evaluate the association between source

Table 2 Description of 45 shared-types (SITs; $n=224$ isolates) which have already been registered in the SITVIT2 or SpolDB4 database and corresponding spoligotyping defined lineages/sublineages starting from a total of 281 M. tuberculosis strains isolated in central Ethiopia

\begin{tabular}{|c|c|c|c|c|c|}
\hline SIT & $\begin{array}{l}\text { Isolates with similar } \\
\text { pattern }\end{array}$ & $\mathrm{CBN}^{*}$ Lineage & $\begin{array}{l}\text { SITVIT2 } \\
\text { Lineage/sublineage }\end{array}$ & Octal number & Binary format \\
\hline 54 & 34 & EA & Manu2 & 777777777763771 & 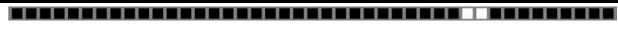 \\
\hline 53 & 43 & EA & $\mathrm{T} 1$ & 777777777760771 & 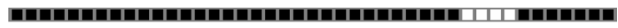 \\
\hline 149 & 37 & EA & T3-ETH & 77700377760771 & ता \\
\hline 37 & 15 & EA & $\mathrm{T} 3$ & 777737777760771 & חד \\
\hline 523 & 7 & IO & Manul & 777777777777771 & inning \\
\hline 817 & 4 & EA & H3-Ural-1 & 777777777420731 & लाता \\
\hline 1690 & 4 & EA & Manu2 & 777777777762771 & 110 \\
\hline 1821 & 9 & $\mathrm{EA}$ & $\mathrm{T}$ & 777347777760771 & \\
\hline 2731 & 5 & EA & Manu2 & 777777777767771 & \\
\hline 52 & 2 & EA & $\mathrm{T} 2$ & 777777777760731 & पा \\
\hline 25 & 7 & EAI & CAS1-Delhi & 703777740003171 & पाता m \\
\hline 26 & 2 & EAI & CAS1-Delhi & 703777740003771 & पाாராणா \\
\hline 121 & 4 & EA & $\mathrm{H} 3$ & 777777775720771 & | \\
\hline 137 & 2 & EA & $\mathrm{X} 2$ & 777776777760601 & IDता \\
\hline 289 & 2 & EAI & CAS1-Delhi & 703777740003571 & $11111110 \mathrm{~mm}$ \\
\hline 47 & 2 & EA & H1 & 777777774020771 & 1110111 \\
\hline 777 & 2 & $\mathrm{EA}$ & H3-Ural-1 & 777777777420771 & पाता \\
\hline 336 & 4 & EA & $\mathrm{X} 1$ & 777776777760731 & ताता \\
\hline 41 & 5 & EA & Turkey & 777777404760771 & तात \\
\hline 119 & 2 & EA & $\mathrm{X} 1$ & 777776777760771 & पाता \\
\hline 1688 & 2 & EA & LAM & 777777403760771 & ताता \\
\hline 50 & 2 & EA & $\mathrm{H} 3$ & 777777777720771 & 1 \\
\hline 1634 & 1 & EA & Manu2 & 777777777723771 & 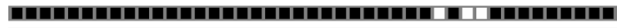 \\
\hline 602 & 1 & EA & $\mathrm{H} 1$ & 777777770000771 & 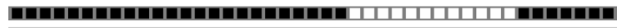 \\
\hline 910 & 1 & M-africanum & AFRI & 700000007177771 & 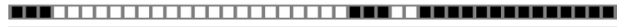 \\
\hline 1590 & 1 & EAI & CAS1-Delhi & 703777340003771 & mand \\
\hline 357 & 1 & EAI & CAS1-Delhi & 703777740000771 & पाताताता \\
\hline 1197 & 1 & EAI & CAS1-Delhi & 703777740203171 & 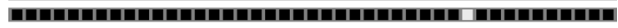 \\
\hline 21 & 1 & EAI & CAS1-Kili & 703377400001771 & 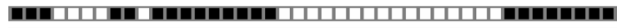 \\
\hline 134 & 1 & EA & $\mathrm{H} 3$ & 777777777720631 & 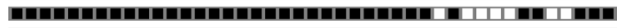 \\
\hline 584 & 1 & $\mathrm{EA}$ & $\mathrm{T} 2$ & 777775777760731 & 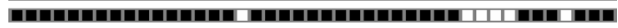 \\
\hline 764 & 1 & EA & $\mathrm{H} 3$ & 777757777720771 & पात \\
\hline 4 & 1 & EA & T1-RUS2 & 000000007760771 & 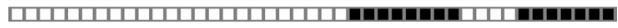 \\
\hline 157 & 1 & EA & $\mathrm{T} 3$ & 777737777760471 & 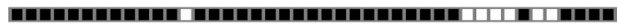 \\
\hline 462 & 1 & EA & $\mathrm{T}$ & 777777777560771 & ता \\
\hline 73 & 1 & EA & $\mathrm{T} 2$ & 777737777760731 & דח \\
\hline 504 & 1 & EA & T3 & 777737737760771 & पात \\
\hline 393 & 1 & EA & $\mathrm{T}$ & 777757777760771 & पा \\
\hline 281 & 1 & EA & $\mathrm{T}$ & 777775777760771 & पात \\
\hline 699 & 1 & EA & $\mathrm{H} 3$ & 677777777720571 & 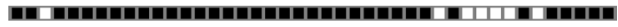 \\
\hline 102 & 1 & EA & $\mathrm{T}$ & 777703777760771 & पाता \\
\hline 966 & 1 & EA & T & 775777777760771 & पाता \\
\hline 93 & 1 & EA & LAM5 & 777737607760771 & जाता \\
\hline 3134 & 4 & EA & $\mathrm{H} 3$ & 777737377720771 & 1101 \\
\hline 3900 & 3 & EA & Manu2 & 777347777763771 & 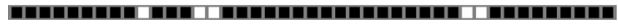 \\
\hline
\end{tabular}

A total of 281 isolates grouped into 90 different spolygotype patterns. Of the 90 spolygtype pattern, 45 were registered in the international database while 45 of the strains were not found in the database. Of these 90 strains, 32 were clustered strains consisting of $223(79.3 \%)$ isolates while 58 strains were found as single isolate. The dominantly found strains were SIT 53, SIT 149, and SIT 54 consisting of 43,37 and 34 isolates, respectively. Classification of the spoligotype patterns using TB-insight RUN TB-Lineage showed that $86.8 \%, 6.4 \%, 5.3 \%, 1.4 \%$ of the isolates belonged to the Euro-American lineage, East-African-Indian, Indo-oceanic and $M$. africanum, respectively. EA Euro-American; EAI East-African Indian; IO Indo-Oceanic; MA M. africanum; MB M. bovis; CBN conformal Bayesian network; KBBN knowledge based Bayesian network 
site of the isolate and clustering status of the isolates. Similarly, Fisher's exact test was used to test of sourcesite is significantly associated with type of major lineage identified as well as with the type of dominant strain. Results were considered statistically significant whenever $p$-value was less than $5 \%$.

\section{Result}

Demographic characteristics of the study participants

Among 338 smear positive sputa samples297 (87.9\%) samples were confirmed as culture positive. A total of $297 M$. tuberculosis isolates were utilized to carry out RD9-PCRand spoligotyping analysis of which 281 gave valid spolygotyping data while the remaining 16 isolates did not give any pattern up on spolygotyping. One hundred thirty (46.2\%) of 281 isolates obtained from female while 151 (53.7\%) were isolated from males. Classification of the study participated on the basis of age showed that $99(35.3 \%)$ were between 18 and 28 years of age, majority $(47.7 \% ; 134 / 281)$ were originated from Woliso and its surroundings (Table 1).
Region of difference (RD) 9-based polymerase chain reaction (PCR). The 281 isolates were analyzed using RD9 PCR and the result indicated that all isolates had intact RD9 implying that all the isolates were M. tuberculosis (Fig. 1).

\section{Spolygotyping result}

Spoligotyping of 281 isolates yielded 90 different spolygotype patterns. Of these, 32 strains were clustered containing of $223(79.3 \%)$ isolates and 58 (20.6\%) were unique strains (Tables 2 and 4). The overall diversity of the isolates was $32.4 \%$. Out of the 90 spolygtype pattern, 45 were registered in the international data base (Table 2) and the remaining 45 were not found in the database (Table 3). The dominantly identified strains were SIT53, SIT149, and SIT 54 consisting of 43 isolates, 37 isolates, 34 isolates, respectively (Table 2). Classification of the spoligotype patterns using TB-insight RUN TBLineage showed that $86.8,6.4,5.3,1.4 \%$ of the isolates belonged to the Euro-American lineage, East-African-

Table 3 Description of 45 orphan strains $(N=57)$ and corresponding spoligotyping defined lineages/sublineages recorded among M. tuberculosis strains starting from a total of 281 M. tuberculosis isolated in central Ethiopia

\begin{tabular}{|c|c|c|c|c|c|}
\hline SIT & $\begin{array}{l}\text { Isolates with similar } \\
\text { pattern }\end{array}$ & $\mathrm{CBN}^{*}$ Lineage & $\begin{array}{l}\text { SITVIT2 } \\
\text { Lineage/sublineage }\end{array}$ & Octal number & Binary format \\
\hline Orphan & 3 & IO & Manul & 7777777777757771 & 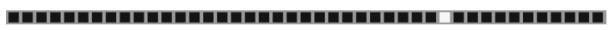 \\
\hline Orphan & 2 & $\mathrm{EA}$ & $\mathrm{T} 3$ & 006737777760771 & 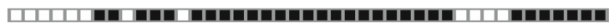 \\
\hline $\begin{array}{l}\text { Orphan } \\
\text { Orphan }\end{array}$ & $\begin{array}{l}2 \\
2\end{array}$ & $\begin{array}{l}\text { EA } \\
\text { EA }\end{array}$ & $\begin{array}{l}\text { T3 } \\
\text { Manu2 }\end{array}$ & $\begin{array}{c}006737737760771 \\
777777777743771\end{array}$ & 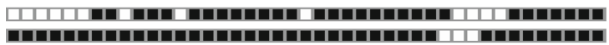 \\
\hline Orphan & 2 & EA & $\mathrm{T} 3$ & 776737677760771 & 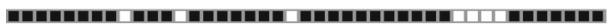 \\
\hline Orphan & 2 & EA & $\mathrm{T} 3$ & 776737777760771 & 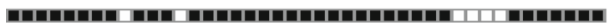 \\
\hline Orphan & 2 & EA & Manu2 & 777747777763771 & 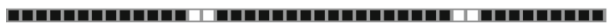 \\
\hline Orphan & 1 & IO & EAI7-BGD2 & 777777770003671 & 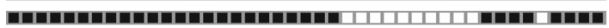 \\
\hline Orphan & 1 & IO & EAI7-BGD2 & 777777770003471 & 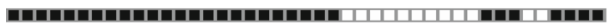 \\
\hline Orphan & 1 & EA & $\mathrm{H} 1$ & 777777760420731 & 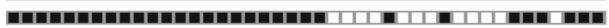 \\
\hline Orphan & 1 & EA & H4-Ural-2 & 501777577420431 & 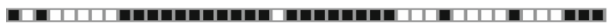 \\
\hline Orphan & 1 & EAI & CAS1-Delhi & 703417740003771 & 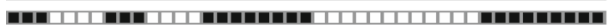 \\
\hline Orphan & 1 & EAI & CAS1-Delhi & 703437740003771 & 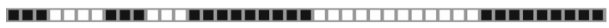 \\
\hline Orrphan & 1 & EAI & CAS1-Kili & 701377404000771 & 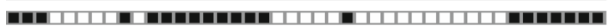 \\
\hline Orphan & 1 & EA & T3-ETH & 777040377760771 & 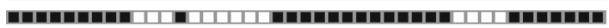 \\
\hline Orphan & 1 & EA & Turkey & 777617404760771 & 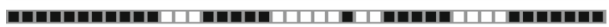 \\
\hline Orrphan & 1 & EA & Turkey & 777377404240771 & 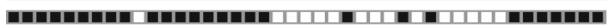 \\
\hline Orphan & 1 & M-africanum & Manu2 & 575347777763661 & 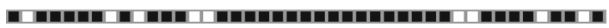 \\
\hline Orphan & 1 & M-africanum & Manu2 & 775347777763671 & 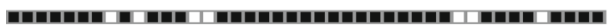 \\
\hline Orphan & 1 & EA & Manu2 & 777777777763671 & 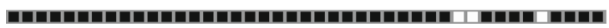 \\
\hline Orphan & 1 & EA & Manu2 & 575347577762671 & 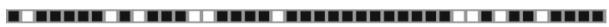 \\
\hline Orphan & 5 & $\mathrm{EA}$ & Manu2 & 777757777763771 & 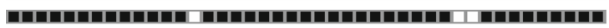 \\
\hline Orphan & 1 & EA & Manu2 & 577347777762671 & 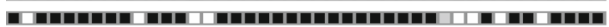 \\
\hline Orphan & 1 & EA & Manu2 & 777777777763760 & 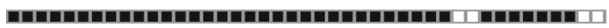 \\
\hline Orphan & 1 & EA & Manu2 & 777747777762771 & 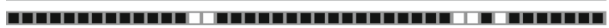 \\
\hline Orphan & 1 & IO & Manu2 & 757777774243471 & 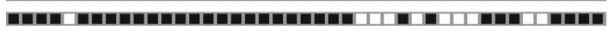 \\
\hline Orphan & 1 & EA & Manu3 & 777347777770371 & 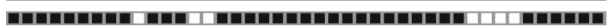 \\
\hline Orphan & 1 & M-africanum & Manu1 & 775347577777771 & 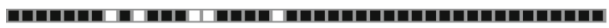 \\
\hline Orphan & 1 & IO & $\mathrm{H} 1$ & 555347430000261 & 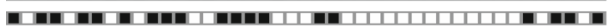 \\
\hline Orphan & 1 & EA & $\mathrm{T}$ & 777345777760671 & 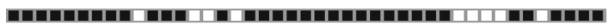 \\
\hline Orphan & 1 & EA & $\mathrm{T}$ & 777347777777771 & 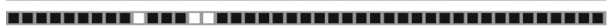 \\
\hline Orphan & 1 & $\mathrm{EA}$ & $\mathrm{T}$ & 777347777760671 & 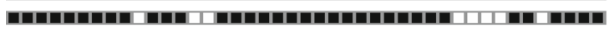 \\
\hline Orphan & 1 & EA & $\mathrm{T}$ & 777245777760771 & 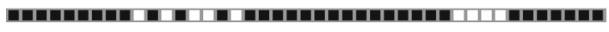 \\
\hline Orphan & 1 & EA & $\mathrm{T}$ & 776737437760771 & 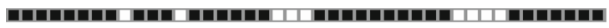 \\
\hline Orphan & 1 & $\mathrm{EA}$ & H3-Ural-1 & 575347777740671 & 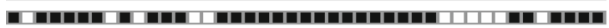 \\
\hline Orphan & 1 & EA & H3-Ural-1 & 773777776000771 & 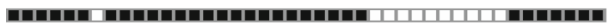 \\
\hline Orphan & 1 & EA & $\mathrm{T} 2$ & 701767777420531 & 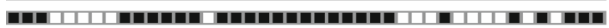 \\
\hline Orphan & 1 & $\mathrm{EA}$ & $\mathrm{T}$ & 777755777760731 & 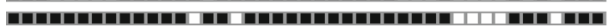 \\
\hline Orphan & 1 & $\mathrm{EA}$ & $\mathrm{T}$ & 577347777740671 & 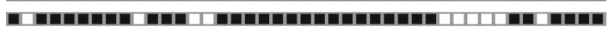 \\
\hline Orphan & 1 & EA & $\mathrm{T}$ & 577347777760771 & 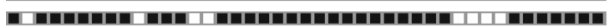 \\
\hline Orphan & 1 & $\mathrm{EA}$ & $\mathrm{T}$ & 577477777760771 & 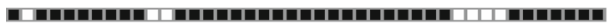 \\
\hline Orphan & 1 & $\mathrm{EA}$ & $\mathrm{T}$ & 777047777760771 & 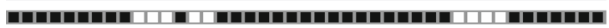 \\
\hline Orphan & 1 & $\mathrm{EA}$ & $\mathrm{T}$ & 557347437740661 & 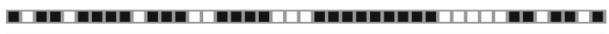 \\
\hline Orphan & 1 & EA & T1-RUS2 & 760002777760771 & 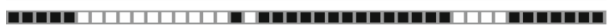 \\
\hline Orphan & 1 & EAI & Manu2 & 777747777763671 & 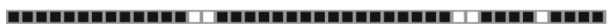 \\
\hline
\end{tabular}

Of the 90 spolygtype pattern, 45 patters consisting of 57 isolates did not match with the patterns available in the database (orphans)

*CBN: conformal Bayesian network; EA EuroAmerican; IO Indo-Oceanic; EAI East African-Indian; MA M. africanum 
Indian, Indo-oceanic and $M$. africanum, respectively (Table 2).

\section{Distribution of strains and lineages in the study area} Of the 281 isolates typed, 134(47.7) were originated from Woliso and its surroundings, with clustering rate of $83.6 \%$ (112/134), 97(34.5) were originated from Fiche with clustering rate of $72.2 \%$ (70/97). The remaining isolates were from Atat town with clustering rate of $14.6 \%(41 / 281)$. This finding did not show statistically significant difference in the proportion of clustering across the three source sites of the isolates $(p$-value = $0.163)$. Similarly, there was not significant association of the source site of the isolate with major lineage identified by CBBN ( $p$-value $=0.877$ ) as well as the type of dominant isolate $(p$-value $=0.109)$. The proportions of occurrence of the dominant lineage (Euro-American) at Woliso, Fitche and Atat towns were 85.5\% (112/131), $89.1 \%(90 / 101)$ and $85.7 \%(42 / 49)$, respectively (Table 4$)$.

\section{Discussion}

In the present study, 281 pulmonary $\mathrm{TB}$ cases were recruited from three towns and their surroundings in central Ethiopia for the isolation and identification of the species and strains of MTC causing pulmonary TB in the central Ethiopia. The isolation of was made from the sputum of patients on LJ medium while RD9-based PCR and spoligotyping used for identification of the isolates at the species and strain levels, respectively. An online tool

Table 4 Distribution of strains and clustering rate in the Study Area $(N=281)$

\begin{tabular}{|c|c|c|c|c|}
\hline \multirow[t]{2}{*}{$\begin{array}{l}\text { Characteristics of the } \\
\text { isolates }\end{array}$} & \multicolumn{3}{|c|}{$\begin{array}{l}\text { Number(\%) of isolates in the } \\
\text { study sites }\end{array}$} & \multirow[t]{2}{*}{$P$-value } \\
\hline & Weliso & Atat & Fiche & \\
\hline Total spolygotyped isolates & $134(47.7)$ & $50(17.8)$ & $97(34.5)$ & \\
\hline \multicolumn{5}{|l|}{ Clustered isolates versus single } \\
\hline Clustered & 112 & 41 & 70 & \multirow[t]{3}{*}{0.163} \\
\hline Single & 22 & 9 & 27 & \\
\hline Clustering rate & $83.6 \%$ & $82 \%$ & $72.2 \%$ & \\
\hline \multicolumn{5}{|l|}{ Major lineage by CBBN } \\
\hline EA & 115 & 43 & 86 & \multirow[t]{4}{*}{0.877} \\
\hline EAl & 10 & 4 & 4 & \\
\hline 10 & 7 & 2 & 6 & \\
\hline MA & 2 & 1 & 1 & \\
\hline \multicolumn{5}{|l|}{ The three dominant Strains } \\
\hline SIT 53 & 19 & 6 & 18 & \multirow[t]{4}{*}{0.109} \\
\hline SIT 149 & 17 & 9 & 11 & \\
\hline SIT 54 & 21 & 8 & 5 & \\
\hline Orphan strains & 21 & 10 & 26 & \\
\hline
\end{tabular}

EA Euro-American, EAl East-African Indian, $1 O$ Indo-Oceanic, MA M. africanum, $M B$ Mbovis, CBN conformal Bayesian network
Run TB-Lineage http://tbinsight.cs.rpi.edu/run_tb_lineage.html was also used for grouping lineages using a conformal Bayesian network (CBN) analysis and sub lineage using knowledge based Bayesian network (KBBN).

Out of the 90 different types of spolygotype patterns, 45 of the patterns which consisting majority of the isolates, matched with the patterns registered in the SITVIT2 database while the remaining 45 patterns did not match with the patterns registered in the SITVIT2 or SpolDB4 database. Similar studies that have been conducted in Ethiopia, and thus all the geographic regions of the country have not been covered and as a result all the circulating strains of $M$. tuberculosis have not yet been registered to the SITVIT2 database. Over $75 \%$ of the isolates were clustered strains with varies sizes of clustering while about a quarter of the isolates were found as unique strains. There are various assumptions with regard to such findings. Although spoligotyping has less discriminatory power in classifying strains, the finding of many isolates clustering in the same pattern could suggest the presence an on-going transmission of $M$. tuberculosis infection in the specific geographic region. On the other hand, the isolation of many unique strains could suggest the introduction of new strains into that specific region and these strains did not spread in that specific geographic region.

Similar to this study, SIT54 was dominantly isolated by earlier studies conducted in the Addis Ababa City (17), central Ethiopia (18) and in eastern central Ethiopia (19). This strain has been mainly reported to the SITVIT2 and SpolDB4 databasefrom South and East Asia, Middle East including Egypt and USA [10, 18]. Another interesting finding in the study was, similar to other studies conducted in Ethiopia earlier [11,13] the ancestor strain SIT 523 was found consisting of good numbers of isolates. SIT 523 is characterized by the presence of all 43 spacers and is the ancestor strain of $M$. tuberculosis. It is less likely that strains keep all the 43 spacers intact for a long duration of time since they have to adapt to different pressures through changing their genetic makeup, and hence it is likely that the presence of 43 spacers intact could also be due to mixed infection, which needs further investigation using more powerful molecular techniques.

The M. tuberculosis isolated by the present study were belonged to four major lineages including the Euro-American, East-African-Indian, Indo-oceanic and the $M$. africanum. The dominant Lineage wasEuroAmerican Lineage consisting $86.8 \%$ of the isolates. This finding is in agreement with the earlier studies $[11,12,19]$ conducted in different regions of Ethiopia. The $2^{\text {nd }}$ and $3^{\text {rd }}$ lineages under which the isolates grouped were EastAfrican-Indian and Indo-oceanic lineage, respectively. Four isolates belonged to $M$. africanum Lineage. $M$. africanum has been reported to be an important cause of 
human TB in the west African countries including Guinea- Bissau [20], the Gambia [21], Sierra Leone [22], Senegal [23], Burkina Faso [24], Cameroon [25], Nigeria [26], and CôteD'Ivoire [27].

The limitation of this study is the use of only spoligotyping for typing of the isolates. Since the discriminatory power of spoligotyping is low, the finding of this study could lead to an overestimation of clustering because of the failure to differentiate between recent transmissions and mixed infections. In contrast to spoligotyping, MIRUVNTR or WGS genotyping allow for a high-resolution and discrimination of the isolates for epidemiological studies and a valid phylogenetic strain classification [28-30].

\section{Conclusion}

The identification of clustered and new strains using spolygotyping in present study could not give conclusive report, as spoligotyping has low discriminatory power. Hence, further characterization of these isolates using MIRU-VNTR or WGS was recommended.

\begin{abstract}
Abbreviations
CBN: Conformal bayesian network; Cl: Confidence intervals; DNA: Deoxonuclic acid; DR: Direct repeats; EA: Euro-American; EAl: East-African -indian; IO: Indo-occenic; IS: Insertion sequence; KBBN: Knowledge based bayesian network; L: Löwenstein —jensen medium; M. tb: Mycobacterium tuberculosis; MA: M.-africanum; MDR-TB: Multi drug resistance tuberculosis; MIRUVNTR: Mycobacterial interspersed repetitive unit-variable number tandem repeat; MTBC: Mycobacterium tuberculosis complex; PCR: Polymerase chain reaction; RD: Regions of difference; SIT: Shared international type; SNPs: Single nucleotide polymorphisms; TB: Tuberculosis; WGS: Whole genome sequence; WHO: World Health Organization
\end{abstract}

\section{Acknowledgments}

This study was jointly funded by the National Institute of Health (NIH, USA) through its H3Africa Consortium Program (Grant Ref. no. U01HG007472-01), Addis Ababa University through its Thematic Research Program, and Hawassa University. We would like to thank the staff member of TB laboratory at St. Lukas, Atat and Fiche Hospitals Hospital without whom this study could not have been completed.

\section{Availability of data and materials}

All of the data is contained within the manuscript

\section{Authors' contributions}

ZB was involved in the design, data collection and laboratory work, and statistical analysis and interpretation of data and drafted the paper; AW was involved in the spoligotyping of the isolates; YM, and GY was involved in critical revision of the paper;GM was involved in interpretation, and critical revision of the paper; GM was involved in statistical analysis, and critical revision of the paper; RP was involved in critical revision of the paper; GA was involved in the design, interpretation, and critical revision of the paper. All authors have read and approved the paper for submission.

\section{Competing interests}

The authors declare that they have no competing interests.

\section{Consent for publication}

Not applicable.

Ethics approval and consent to participate

Ethical clearance was obtained from Ethical Review Board of Natural Science, Addis Ababa University, Ethiopia (Ref. No. CNSDO/379/07/15). In addition, the purpose of the study was explained to all enrolled subjects in simple terms, and written informed consents were obtained from each of the study participants.

\section{Author details}

${ }^{1}$ Aklilu Lemma Institute of Pathobiology, Addis Ababa University, P.O. Box 1176, Addis Ababa, Ethiopia. ${ }^{2}$ College of Natural Sciences, Microbial, Cellular and Molecular Biology Department, Addis Ababa University, P.O. Box 1176, Addis Ababa, Ethiopia. ${ }^{3}$ College of Natural Science and Computational Science, Department of Biology, Hawassa University, P.O. Box 05, Hawassa, Ethiopia. ${ }^{4}$ College of Veterinary Medicine and Agriculture, Addis Ababa University, P.O. Box 34, DebreZeit, Ethiopia. ${ }^{5}$ College of Health Sciences, Addis Ababa University, P.O. Box 1176, Addis Ababa, Ethiopia. ${ }^{6}$ J.Craig Venter Institute, 9704 Medical Center Drive, Rockville, MD, USA.

Received: 6 December 2015 Accepted: 15 February 2017

Published online: 01 March 2017

\section{References}

1. WHO. Global tuberculosis report. Geneva, Switzerland: WHO; 2015.

2 Federal Ministry of Health. Guideline for program and clinical management of drug resistant tuberculosis. Ethiopia. Firstth ed. Addis Ababa: FMOH; 2009.

3 Van Soolingen D. Utility of molecular epidemiology of tuberculosis. Eur Respir J. 1998;11:795-7.

4 Paranjothy S, Eisenhut M, Lilley M, Bracebridge S, Abubakar I. Extensive transmission of Mycobacterium tuberculosis from 9 years old child with pulmonary tuberculosis and negative sputum smear. BMJ. 2008;337:1184.

5 Tostmann AS, Kik V, Kalisvaart NA, Sebek MM, Verver S. Tuberculosis transmission by patients with smear-negative pulmonary tuberculosis in a large cohort in the Netherlands. Clin Infect Dis. 2008;47:1135-42.

6 Dobler CC, Marks GB, Simpson SE, Crawford AB. Recurrence of tuberculosis at a Sydney chest clinic between 1994 and 2006: reactivation or reinfection? Med J Aust. 2008;188:153-5.

7 Allix C, Supply P, Fauville-Dufaux M. Utility of fast mycobacterial interspersed repetitive unit-variable number tandem repeat genotyping in clinical mycobacteriological analysis. Clin Infect Dis. 2004;39:783-9.

8 Mathema B, Kurepina NE, Bifani PJ, Kreiswirth BN. Molecular epidemiology of tuberculosis: current insights. Clin Microbiol Rev. 2006;19:658-85.

9 Allix-Beguec C, Fauville-Dufaux M, Stoffels K, Ommeslag D, Walravens K. Importance of identifying Mycobacterium bovis as a causative agent of human tuberculosis. Eur Respir J. 2010;35:692-4.

10 SITVIT1Database. [http://www.pasteur-guadeloupe.fr:8081/SITVITDemo/]. Accessed 10 Sept 2012.

11 Diriba B, Berkessa T, Mamo G, Tedla Y, Ameni G. Spoligotyping of multidrug-resistant Mycobacterium tuberculosis isolates in Ethiopia. Int J Tuberc Lung Dis. 2013;17(2):246-50.

12 Garedew L, Mihret A, Mamo G, Abebe T, Firdessa R, Bekele Y, Ameni G. Strain diversity of mycobacteria isolated from pulmonary tuberculosis patients at DebreBirhan Hospital, Ethiopia. Int J Tuberc Lung Dis. 2013;17(8):1076-81.

13 Mulugeta B, Ameni G, Bjune G, Couvin D, Rastogi N, and Abebe. Strain Diversity of Mycobacterium tuberculosis Isolates from Pulmonary Tuberculosis Patients in Afar Pastoral Region of Ethiopia. Biomed Res Int. 2014.

14 WHO. Laboratory services in tuberculosis control: culture. Part III. Geneva, Switzerland: WHO; 1998.

15 Yates M, Drobniewski F, Wilson S. Evaluation of a rapid PCR-based epidemiological typing method for routine studies of Mycobacterium tuberculosis. J Clin Microbiol. 2002;40:712-4

16 Parsons ML, Brosch R, Stewart T, et al. Rapid and simple approach for identification of Mycobacterium tuberculosis complex isolates by PCR-based genomic deletion analysis. J Clin Microbiol. 2002;40:2339-45.

17 Kamerbeek J, Schouls L, Kolk A, van Agterveld M, van Soolingen D, et al. Simultaneous detection and strain differentiation of Mycobacterium tuberculosis for diagnosis and epidemiology. J Clin Microbiol. 1997;35(4):907-14.

18 Brudey K, Driscoll JR, Rigouts L, Prodinger WM, Gori A, et al. Mycobacterium tuberculosis complex genetic diversity: mining the fourth international spoligotyping database (SpolDB4) for classification population genetics and epidemiology. BMC Microbiol. 2006;6:23.

19 Debebe T, Admassu A, Mamo G, Ameni G. Molecular characterization of Mycobacterium tuberculosis isolated from pulmonary tuberculosis patients in FelegeHiwot referral hospital, northwest Ethiopia. J Microbial. 2014;47(4): 333-8. 
20 Källenius G, Koivula T, Ghebremichael S, Hoffner S, Norberg R, et al. Evolution and clonal traits of Mycobacterium tuberculosis complex in Guinea-Bissau. J Clin Microbiol. 1999;37(12):3872-8.

21 Jong B, Hill P, Brookes R, Gagneux S, Jeffries D, Otu J, et al. Mycobacterium africanum elicits an attenuated $T$ cell response to early secreted antigenic target, $6 \mathrm{kDa}$, in patients with tuberculosis and their household contacts. J Infect Dis. 2006;193(9):1279-86.

22 Homolka S, Post E, Oberhauser B, George A, Westman L, Dafae F, RüschGerdes $S$, Niemann S. High genetic diversity among Mycobacterium tuberculosis complex strains from Sierra Leone. BMC Microbiol. 2008:8:103.

23 Viana-Niero C, Gutierrez C, Sola C, Filliol I, Boulahbal F, Vincent V, Rastogi N. Genetic diversity of Mycobacterium africanum clinical isolates based on IS6110-restriction fragment length polymorphism analysis, spoligotyping, and variable number of tandem DNA repeats. J Clin Microbiol. 2001;39(1):57-65.

24 Ledru S, Cauchoix B, Yaméogo M, Zoubga A, Lamandé-Chiron J, Portaels F, Chiron J. Impact of short-course therapy on tuberculosis drug resistancein South-West Burkina Faso. Tuber Lung Dis. 1996;77(5):429-36.

25 Niobe-Eyangoh S, Kuaban C, Sorlin P, Cunin P, Thonnon J, Sola C, Rastogi N, Vincent V, Gutierrez M. Genetic biodiversity of Mycobacterium tuberculosis complex strains from patients with pulmonary tuberculosis in Cameroon. J Clin Microbiol. 2003;41(6):2547-53.

26 Cadmus S, Palmer S, Okker M, Dale J, Gover K, Smith N, Jahans K, Hewinson $\mathrm{R}$, Gordon $\mathrm{S}$. Molecular analysis of human and bovine tubercle bacilli from a local setting in Nigeria. J Clin Microbiol. 2006;44(1):29-34.

27 Niemann S, Kubica T, Bange F, Adjei O, Browne E, et al. The species Mycobacterium africanum in the light of new molecular markers. J Clin Microbiol. 2004;42(9):3958-62.

28 Supply P, Allix C, Lesjean S, Cardoso-Oelemann M, Rüsch-Gerdes S. Proposal for standardization of optimized mycobacterial interspersed repetitive unitvariable- number tandem repeat typing of Mycobacterium tuberculosis. J Clin Microbiol. 2006;44:4498-510.

29 Cowan L, Diem L, Monson T, Wand P, Temporado D, Oemig T, Crawford J. Evaluation of a two-step approach for large-scale, prospective genotyping of Mycobacterium tuberculosis isolates in the United States. J Clin Microbiol. 2005;43:688-95.

30 Comas I, Coscolla M, Luo T, Borrell S, Holt K, Kato-Maeda M. Out-of-Africa migration and Neolithic co expansion of Mycobacterium tuberculosis with modern humans. Nat Genet. 2013:45:1176-82.

\section{Submit your next manuscript to BioMed Central and we will help you at every step:}

- We accept pre-submission inquiries

- Our selector tool helps you to find the most relevant journal

- We provide round the clock customer support

- Convenient online submission

- Thorough peer review

- Inclusion in PubMed and all major indexing services

- Maximum visibility for your research

Submit your manuscript at www.biomedcentral.com/submit

) Biomed Central 